\title{
Analytic Solutions of a Two-Fluid Hydrodynamic Model
}

\author{
Imre Ferenc Barna ${ }^{a}$ and László Mátyás ${ }^{b}$ \\ ${ }^{a}$ Wigner Research Center for Physics \\ Konkoly-Thege Miklós út 29-33, 1121 Budapest, Hungary \\ ${ }^{b}$ Department of Bioengineering, Faculty of Economics, Socio-Human Sciences \\ and Engineering \\ Sapientia Hungarian University of Transylvania Libertătii sq. 1, 530104 \\ Miercurea Ciuc, Romania \\ E-mail(corresp.): barna.imre@wigner.hu \\ E-mail: matyaslaszlo@uni.sapientia.ro
}

Received September 29, 2020; revised September 16, 2021; accepted September 17, 2021

\begin{abstract}
We investigate a one dimensional flow described with the non-compressible coupled Euler and non-compressible Navier-Stokes equations in the Cartesian coordinate system. We couple the two fluids through the continuity equation where different void fractions can be considered. The well-known self-similar Ansatz was applied and analytic solutions were derived for both velocity and pressure field as well.
\end{abstract}

Keywords: self-similar solution, two-fluid model.

AMS Subject Classification: 33B20; 35Q30.

\section{Introduction}

There is no need to prove the evidence that investigation of hydrodynamic systems have crucial importance for human society and civilization. The second evidence is that, there are almost infinite variety of flows in nature or in engineering, some of them are viscous and some of them can be considered as ideal fluids. We may narrow the flow frames to systems of multi-phase flows where a liquid flows together with its vapor or with other non-condensible gas (a good example is water-air flow). Multi-phase flows are relevant in numerous fields like nuclear industry, hydrology chemistry or petrochemistry. We may refine

Copyright (c) 2021 The Author(s). Published by Vilnius Gediminas Technical University This is an Open Access article distributed under the terms of the Creative Commons Attribution License (http://creativecommons.org/licenses/by/4.0/), which permits unrestricted use, distribution, and reproduction in any medium, provided the original author and source are credited. 
multi-phase flows - where large temperature gradient is present between the two phases of the media (eg. water and steam) making condensation and boiling process possible - calling it thermal-hydraulics. The most intensively studied material is the water-steam system which is relevant for nuclear industry. In the last fifty years it's literature become enormous, without completeness we just mention some relevant monographs $[13,14,16]$. The readers who are more concentrated to the mathematics of the models should read [18,24] all models are based on gas dynamics. A decade ago we investigated the steam condensation induced water hammer (CIWH), which is the most complex two-phase flow including explosion-like condensation of hot steam to warm water. We could theoretically explain our experimentally measured 130 bar over pressure peaks which have $2 \mathrm{~ms}$ pulse width [6] for the first time. The original model was developed by Tiselj and Petelin [25]. Ultimately, we investigated a proton beam induced two-phase flow pressure waves in mercury [7] which are relevant for spallation neutron sources. Numerous thermal hydraulic models exist for two-phase flows which contain from two up to seven coupled partial differential equations (PDE) for mass, momenta and energy conservation. Unfortunately all such models are for one spatial dimension only. In the physics of multi-phase flows one of the most relevant dynamical variable is the void fraction (usually noted with $\alpha$ ) which describes the volume ratios (usually steam to water) of the two fluids in a given space point at a given time. In our next model we also use the void fraction but just as a free coupling parameter between the two velocities of the two fluids.

The second relevant field of fluid dynamics is two-fluid flows. Of course these two systems are not the same the questions and problems are different, but are not distinct as well.

We may define these systems where two liquids flow together with different physical parameters like, density, viscosity and thermal properties, but both should be in the same phase (gas-gas or liquid-liquid). The question of superfluidity can be handled with such two-fluid models. At very low temperatures some special viscous fluids become superfluids which means, that they loose their internal viscosity. All technical and historical details can be found in numerous textbooks $[15,21,22]$.

The original idea how to solve the superfluidity problem, namely to couple viscous and inviscid fluids came from Landau in 1941 [17]. Interesting aspects on the evolution of the two-fluid model related to superfluidity one can find in [1], where both the Tisza [26,27] and the Landau model [17] is mentioned. Later the idea of superfluidity became quite widely spread e.g. in nuclear [19], high energy or astrophysics to explain exotic phases of matter [28].

It is evident from physical considerations that there are numerous ways to couple the ideal and viscous fluids together, this is done usually by their densities. For compressible fluids the equation of state (EOS) could also couple the dynamical equations of the two fluids via their common pressure. Worth to mention, that in our simple presented model we just consider an incompressible continuity equation where the velocities of the two phases are weighted with their void fraction. This is the most simple model to couple an Euler to a Navies-Stokes equation, of course in the future we want to develop our 
description to more an more realistic one.

In this study we investigate the self-similar Ansatz $[2,23,29]$ applied the two-fluid model which describes physically relevant disperse or dissipate solutions. This study is organically linked to our long-term program in which we systematically goes over fundamental hydrodynamic systems and analyze physically relevant self-similar and traveling wave solutions. Till now we published about half a dozen papers $[3,4,5,8,9,10]$ and a book chapter [12] in this field. Due to our knowledge there is no self-similar solution known and analyzed for time-dependent two-fluid models.

\section{Theory and results}

Let's start with the following PDE flow system of

$$
\begin{aligned}
& a \frac{\partial v_{1}}{\partial x}+(1-a) \frac{\partial v_{2}}{\partial x}=0 \\
& \frac{\partial v_{1}}{\partial t}+v_{1} \frac{\partial v_{1}}{\partial x}=-\frac{1}{\rho_{1}} \frac{\partial p}{\partial x}, \\
& \frac{\partial v_{2}}{\partial t}+v_{2} \frac{\partial v_{2}}{\partial x}=-\frac{1}{\rho_{2}} \frac{\partial p}{\partial x}+\nu \frac{\partial^{2} v_{2}}{\partial x^{2}},
\end{aligned}
$$

where the dynamical variables are the two fluid velocities $v_{1}(x, t), v_{2}(x, t)$ and the common pressure $p(x, t)$ there are four additional physical constants $a, \rho_{1}$, $\rho_{2}$ and $\nu$ which are the void fraction, the two fluid densities and the viscosity of the second fluid.

We apply the following self-similar Ansatz for the variables:

$$
v_{1}(x, t)=t^{-\alpha} f(\eta), \quad v_{2}(x, t)=t^{-\gamma} g(\eta), \quad p(x, t)=t^{-\delta} h(\eta)
$$

with the new variable $\eta=\frac{x}{t^{\beta}}$. All the exponents $\alpha, \beta, \gamma, \delta$ are real numbers. (Solutions with integer exponents are called self-similar solutions of the first kind, non-integer exponents generate self-similar solutions of the second kind.) The shape functions $f, g, h$ could be any continuous functions with existing first and second continuous derivatives and will be evaluated later on. The logic, the physical and geometrical interpretation of the Ansatz were exhaustively analyzed in all our former publications $[3,4,9,10,12]$ therefore we neglect it.

To have consistent coupled ordinary differential equation system (ODEs) for the shape functions the exponents have to have the following values of

$$
\alpha=\beta=\gamma=1 / 2, \quad \delta=1
$$

Such fixed exponents were found for the multi-dimensional incompressible Navier-Stokes equations [3] as well. This means that the pressure field has a stronger decay than the velocity fields.

The following unequivocal ordinary differential equation (ODE) system can be obtained

$$
a f^{\prime}+(1-a) g^{\prime}=0
$$




$$
\begin{aligned}
& -\frac{1}{2} f-\frac{1}{2} \eta f^{\prime}+f f^{\prime}=-\frac{h^{\prime}}{\rho_{1}}, \\
& -\frac{1}{2} g-\frac{1}{2} \eta g^{\prime}+g g^{\prime}=-\frac{h^{\prime}}{\rho_{2}}+\nu g^{\prime \prime} .
\end{aligned}
$$

Note, that unlike the tested systems so far, all three equations are total derivatives and can be integrated once. (All three equations are conservation equations, so this statement is straightforward, however our decade-long experience tells that usually only the first - the continuity - equation has such property.) After some ordinary algebraic steps - which means substituting one equation into the other, and sorting the terms - we get the final ODE for the velocity shape function of the form of

$$
\begin{gathered}
-\nu \rho_{2} g^{\prime}-\frac{\eta g \rho_{2}}{2}+\frac{g^{2} \rho_{2}}{2}-c_{3} \rho_{2}=-\frac{\eta \rho_{1}}{2}\left[\frac{(a-1) g+c_{1}}{a}\right] \\
+\frac{\rho_{1}}{2}\left(\frac{\left[\{a-1\} g+c_{1}\right]}{a}\right)-c_{2} \rho_{2},
\end{gathered}
$$

where $c_{1}, c_{2}$ and $c_{3}$ are the three integration constants of $(2.1)-(2.3)$.

All our investigated systems have a kind of hierarchy, due to the nonlinearity of the variables and the asymmetric form, there is always a prior quantity which would be evaluated first. As we see, now the viscous velocity field $g(\eta)$ comes first. (There is a general impenetrable many page long complex analytic solution available for (2.4) for the most general $c_{1}, c_{2}, c_{3} \neq 0$ case consisting large number of Kummer $\mathrm{M}$ and Kummer $\mathrm{U}$ functions according to the symbolic Computation Software Maple 12, which we skip now.) All these three integration constants mean just general shifts in the solution function. For $c_{1}=c_{2}=c_{3}=0$ case the shape function of the velocity field is still compact enough

$$
\begin{aligned}
g= & 2 \nu \sqrt{-\frac{\rho_{1} a-\rho_{1}-\rho_{2} a}{\nu \rho_{2} a}} e^{\frac{1}{4} \eta\left(-\eta \rho_{2} a+\eta \rho_{1} a-\eta \rho_{1}-2 \rho_{1} a+2 \rho_{1}\right) / \nu \rho_{2} a} \\
\times & \left(2 \sqrt{-\frac{\rho_{1} a-\rho_{1}-\rho_{2} a}{\nu \rho_{2} a}} \nu c_{4}+\sqrt{\pi} e^{-\frac{1}{4} \frac{\rho_{1}^{2}(a-1)^{2}}{\nu \rho_{2} a\left(\rho_{1} a-\rho_{1}-\rho_{2} a\right)}}\right. \\
& \left.\times \operatorname{erf}\left(\frac{1-\eta \rho_{2} a+\eta \rho_{1} a-\eta \rho_{1}-\rho_{1} a+\rho_{1}}{2}\right)\right)^{-1},
\end{aligned}
$$

where the $\operatorname{erf}$ is the usual error function [20]. In general the error function may also appear as a solution of diffusion equation [11] or the KPZ equation [4] in certain cases.

The velocity field of the ideal fluid is the following

$$
f=\frac{a-1}{a} g+\frac{c_{4}}{a},
$$

due to the one dimensional property of the model the velocity field of the ideal fluid is just scaled by the parameter $a$. Note, that both velocity fields behave similarly and both contain error functions. 


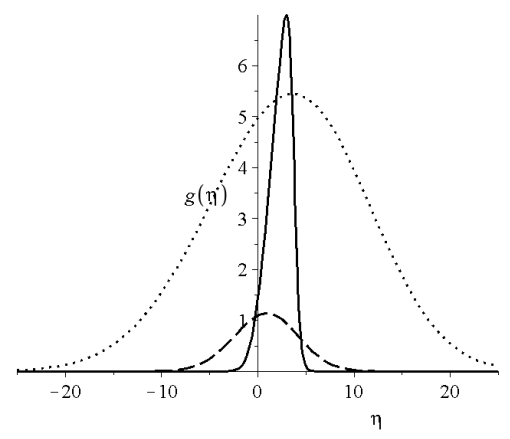

Figure 1. The graphs of Equation (2.5) for various parameter sets. The solid curve is for $c_{4}=0.5, a=0.5, \nu=1.6, \rho_{1}=2, \rho_{2}=1$ the dotted line is for $c_{4}=0.9, a=0.27, \nu=10.4, \rho_{1}=2, \rho_{2}=6$ and the dashed curve is for $c_{4}=0.2, a=0.27, \nu=40, \rho_{1}=2, \rho_{2}=20$, respectively.

Figure 1 shows the shape function of viscous velocity field for various physical parameters.

We may say that the functions are zero at large negative arguments, then have a finite value in the origin then have a not-so-pointed maxima and a quick decay to zero at large positive arguments. Different viscosity values, density rates or void fractions cannot modify the general features of the solution.

It is worth noting here, that the well-known Rayleigh-Bénard convection model - which couples the two dimensional Navier-Stokes equation to heat conduction - has a similar solution as well [9]. All the velocity, pressure and temperature fields can be expressed with an error function, too. Very roughly, we may say that a solution expressed with error functions is almost Gaussian, which means a very sharp temporal and spatial decay.

For the sake of completeness we have to mention an additional property of the velocity shape function, for a small numerical value of $c_{4}$ the denominator can be zero which means a singularity for the shape function and for the velocity field as well. We can see from Equation (2.6) that the shape function of the pressure field has the same singularity for $c_{5}=0$ as well.

Figure 2 presents the final velocity distribution $v_{1}(x, t)$ of the viscous fluid. Due to the extra $t^{-\frac{1}{2}}$ prefactor the distribution has an extreme quick time decay. It is also interesting, that for a given spatial coordinate, if $t \rightarrow \infty$, $\eta \rightarrow 0$, then $g$ has a finite value. This means, that for sufficiently large times at a given $x$, we have

$$
v_{2} \simeq g(0) / t^{1 / 2}
$$

The situation is similar for the other velocity field. For large times at a given spatial coordinate, a finite $g(0)$ implies a finite $f(0)$. Correspondingly we have

$$
v_{1} \simeq f(0) / t^{1 / 2}
$$

As one can see, the two velocity field decay in the same rhythm for large times. The pressure field is a bit more complicated, but can be evaluated without 


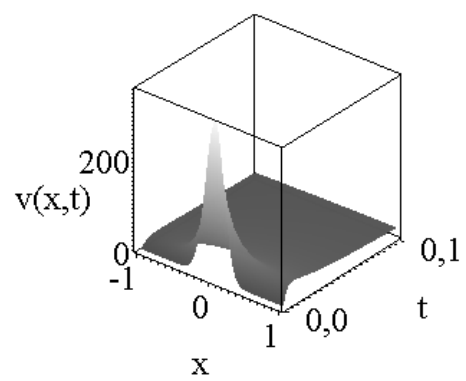

Figure 2. The final velocity distribution of $v_{1}=\frac{1}{t^{\frac{1}{2}}} g\left(\frac{x}{t^{\frac{1}{2}}}\right)$ for the paramter set of $c_{4}=0.2, a=0.27, \nu=40, \rho_{1}=2, \rho_{2}=20$.

integration via

$$
h=-\frac{\rho_{1} f^{2}}{2}+\frac{\rho_{1} \eta f}{2}+c_{5} \rho_{1}
$$

Figure 3 shows the pressure shape functions for three different parameter sets. The functions have a bit more complicated structure than the velocity distributions, there is a local minima and maxima.

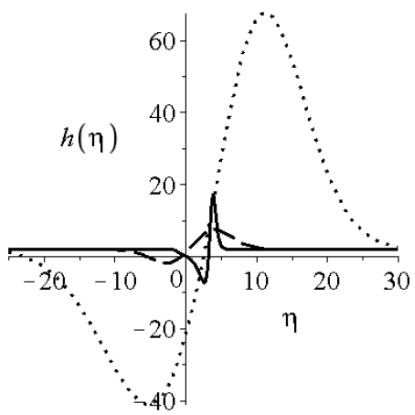

Figure 3. The shape function of the pressure (2.6) for the three parameter sets. The solid curve is for $c_{4}=0.5, a=0.5, \nu=1.6, \rho_{1}=2, \rho_{2}=1$ the dotted line is for $c_{4}=0.9, a=0.27, \nu=10.4, \rho_{1}=2, \rho_{2}=6$ and the dashed curve is for $c_{4}=0.2, a=0.27, \nu=40, \rho_{1}=2, \rho_{2}=20$, respectively.

Our last Figure 4 shows the pressure distribution function. Similar to the shape function, it has a very sharp local minima and maxima as well. Due to the different exponent $\delta=1$ the pressure field has a much quicker decay than the velocity field, which is long known for us [3]. 


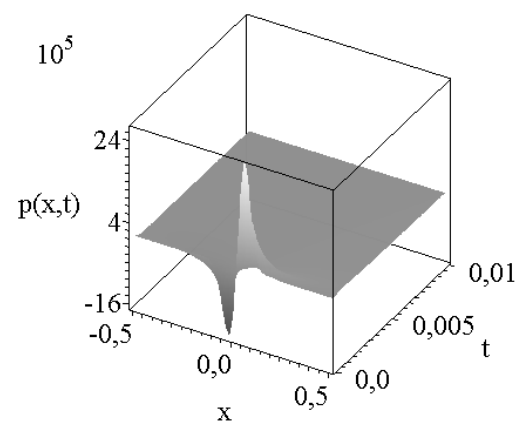

Figure 4. The final pressure distribution of (2.6) $p=\frac{1}{t^{1}} h\left(x / t^{0.5}\right)$ for the parameter set of $c_{4}=0.2, c_{5}=0, a=0.27, \nu=40, \rho_{1}=2, \rho_{2}=20$. Note, that the pressure in (2.6) is defined up to a constant value. If one wants to avoid negative pressures, then an appropriate constant pressure should be added to the values presented above.

\section{Conclusions}

After an introduction of multi-phase and multi-fluid flows and their role in superconductivity we presented the probable most simple one dimensional twofluid model. An incompressible Euler and Navier-Stokes equations are coupled together via the continuity equations where the divergences of the fluid fields were scaled with the void fraction. The self-similar Ansatz was introduced and applied to all dynamical variables of the problem. A coupled ODE system was evaluated for the velocity and pressure fields. Finally, an analytic solution for the velocity field was derived which contains the error function. The pressure field was easily calculated from the velocity field as well. Parameter studies were done for both dynamical variables. Further work is in progress to enhance the complexity of our present model. There are many natural ways possible, the first one is to use more realistic coupling between the fluids via the corresponding densities. In the second way we may consider that one of both fluids are compressible with a common pressure, and the EOS would make the coupling. As a third way heat conduction could be included as well. It is clear that the horizon of the presented problem will be quite wide.

\section{Acknowledgements}

One of us (I.F. Barna) was supported by the NKFIH, the Hungarian National Research Development and Innovation Office. This work was supported by project no. 129257 implemented with the support provided from the National Research, Development and Innovation Fund of Hungary, financed under the K18 funding scheme. The described study was carried out as part of the EFOP3.6.1-16-00011 Younger and Renewing University Innovative Knowledge City institutional development of the University of Miskolc aiming at intelligent specialization project implemented in the framework of the Szechenyi 2020 
program. The realization of this project is supported by the European Union, co-financed by the European Social Fund. Finally, the authors dedicate this paper to their parents.

\section{References}

[1] S. Balibar. Laszlo Tisza and the two-fluid model of superfluidity. Comptes Rendus Physique, 18(9):586, 2017. https://doi.org/10.1016/j.crhy.2017.10.016.

[2] G.I. Baraneblatt. Similarity, Self-Similarity, and Intermediate Asymptotics. Consultants Bureau, New York, 1979.

[3] I.F. Barna. Self-similar solutions of three-dimensional Navier-Stokes equation. Communications in Theoretical Physics, 56(4):745-750, 2011. https://doi.org/10.1088/0253-6102/56/4/25.

[4] I.F. Barna, G. Bognár, M. Guedda, L. Mátyás and K. Hriczó. Analytic selfsimilar solutions of the Kardar-Parisi-Zhang interface growing equation with various noise terms. Mathematical Modelling and Analysis, 24(2):241-256, 2020. https://doi.org/10.3846/mma.2020.10459.

[5] I.F. Barna, G. Bognár and K. Hriczó. Self-similar analytic solution of the two-dimensional Navier-Stokes equation with a non-Newtonian type of viscosity. Mathematical Modelling and Analysis, 21(1):83-94, 2016. https://doi.org/10.3846/13926292.2016.1136901.

[6] I.F. Barna, A.R. Imre, G. Baranyai and Gy. Ézsol. Experimental and theoretical study of steam condensation induced water hammer phenomena. Nuclear Engineering and Design, 240:146-150, 2010. https://doi.org/10.1016/j.nucengdes.2009.09.027.

[7] I.F. Barna, A.R. Imre, L. Rosta and F.Mezei. Two-phase flow model for energetic proton beam induced pressure waves in mercury target systems in the planned European Spallation Source. The European Physical Journal B, 66(4):419-426, 2008. https://doi.org/10.1140/epjb/e2008-00444-x.

[8] I.F. Barna and R. Kersner. Heat conduction: a telegraph-type model with self-similar behavior of solutions. Journal of Physics A: Mathematical and Theoretical, 43(37):375210, aug 2010. https://doi.org/10.1088/1751$8113 / 43 / 37 / 375210$.

[9] I.F. Barna and L. Mátyás. Analytic self-similar solutions of the OberbeckBoussinesq equations. Chaos, Solitons \& Fractals, 78:249-255, 2015. ISSN 0960-0779. https://doi.org/10.1016/j.chaos.2015.08.002.

[10] I.F. Barna, L. Mátyás and M.A. Pocsai. Self-similar analysis of a viscous heated Oberbeck-Boussinesq flow system. Fluid Dynamics Research, 52(1):015515, mar 2020. https://doi.org/10.1088/1873-7005/ab720c.

[11] G.W. Bluman and J.D. Cole. The general similarity solution of the heat equation. Indiana University Mathematics Journal, 18(11):1025-1042, 1969. https://doi.org/10.1512/iumj.1969.18.18074.

[12] D. Campos. Handbook on Navier-Stokes Equations, Theory 83 Applied Analysis. Nova Publishers, New York, 2017.

[13] C.T. Crowe. Multiphase Flow Handbook. CRC Taylor and Francis, 2006. https://doi.org/10.1201/9781420040470. 
[14] M. Ishii and T. Hibiki. Thermo-Fluid Dynamics of Two-Phase Flow. Springer, New York, 2011. https://doi.org/10.1007/978-1-4419-7985-8.

[15] I.M. Khalatnikov. An Introduction to the Theory of Superfluidity. Westview Press, Boulder Colorado, 2000.

[16] N.I. Kolev. Multiphase Flow Dynamics. Springer, 2006.

[17] L. Landau. Theory of the superfluidity of helium II. Physical review journals archieve, 60:356-358, Aug 1941. https://doi.org/10.1103/PhysRev.60.356.

[18] R. Menikoff and B.J. Plohr. The Riemann problem for fluid flow of real materials. Reviews of modern physics, 61:75-130, Jan 1989. https://doi.org/10.1103/RevModPhys.61.75.

[19] A.B. Migdal. Superfluidity and the moments of inertia of nuclei. Nuclear Physics, 13(5):655- 674, 1959. ISSN 0029-5582. https://doi.org/10.1016/00295582(59)90264-0.

[20] F.W.J. Olver, D.W. Lozier, R.F. Boisvert and C.W Clark. The NIST handbook of mathematical functions. Cambridge University Press, New York, NY, 2010.

[21] L. Pitaevskii and S. Stringari. Bose-Einstein Condensation and Superfluidity. Oxford Science Publications, 2015. https://doi.org/10.1093/acprof:oso/9780198758884.001.0001.

[22] A. Scmidth. Introduction to Superfluidity. Springer, Cham, 2015. https://doi.org/10.1007/978-3-319-07947-9.

[23] L.I. Sedov. Similarity and Dimensional Methods in Mechanics. Mechanics CRC Press, 1993.

[24] H.B. Stewart and B. Wendroff. Two-phase flow: Models and methods. Journal of Computational Physics, 56(3):363-409, 1984. https://doi.org/10.1016/00219991(84)90103-7.

[25] I. Tiselj and S. Petelin. Modelling of two-phase flow with second-order accurate scheme. Journal of Computational Physics, 136(2):503-521, 1997. https://doi.org/10.1006/jcph.1997.5778.

[26] L. Tisza. Green's theory of liquid helium. Nature, 163:102-103, 1938. https://doi.org/10.1038/163102a0.

[27] L. Tisza. The theory of liquid helium. Physical review journals archive, 72:838854, Nov 1947. https://doi.org/10.1103/PhysRev.72.838.

[28] G.E. Volovik. The Universe in a Helium Droplet. Clarendon Press, 2003. https://doi.org/10.1093/acprof:oso/9780199564842.001.0001.

[29] Ya.B. Zel'dovich and Yu.P. Raizer. Physics of Shock Waves and High Temperature Hydrodynamic Phenomena. Academic Press, New York, 1966. 